\title{
Avaliação do Programa Bolsa Família (PBF) na segurança alimentar e nutricional das famílias beneficiadas, Vitória da Conquista, BA
}

\author{
Evaluation of the Bolsa Família Program (PBF) on food and nutritional security \\ in Beneficiary families, Vitória da Conquista, BA

\section{Évaluation du Programme Bolsa Familia (PBF) sur la sécurité alimentaire et la nutrition des familles bénéficiaires, Vitória da Conquista, $B A$}

\section{Evaluación del Programa Bolsa Familia (PBF) en la seguridad alimentaria y nutricional de las familias beneficiadas, Vitória da Conquista, $B A$}

\author{
André Souza Suzart ${ }^{1}$ \\ Aldo Pacheco Ferreira ${ }^{2}$ \\ Recebido em 23/06/2017; revisado e aprovado em 14/11/2017; aceito em 22/11/2017 \\ DOI: http://dx.doi.org/10.20435/inter.v19i3.1636
}

\begin{abstract}
Resumo: Este trabalho discorre sobre a análise do Programa Bolsa Família na segurança alimentar e nutricional das famílias beneficiadas do município de Vitória da Conquista, BA. A partir da aplicação de formulários semiestruturados com os beneficiários e das observações de campo, o diagnóstico destacou a necessidade de ações de responsabilidade social e de políticas públicas como garantia da segurança alimentar e nutricional das famílias que se utilizam desse programa social.

Palavras-chave: segurança alimentar e nutricional; pobreza; impacto no estado de saúde; saúde pública; política nutricional.
\end{abstract}

Abstract: This study discusses the analysis of the Family Grant Program on the aspects of security and nutrition in beneficiaries of the city of Vitória da Conquista, BA. From the application form semistructured with beneficiaries and field observations, the diagnosis emphasized the need for social responsibility and public policy as ensuring food and nutrition security for families who use this social program.

Keywords: food and nutrition security; poverty; health evaluation; public health; nutrition policy.

Résumé: Cette étude évalue la Bolsa Familia sur les aspects de la sécurité et de la nutrition chez les bénéficiaires de la ville de Vitória da Conquista, BA. Des formulaires de demande de semi-structurés avec les bénéfi ciaires et les observations de terrain, le diagnostic a mis l'accent sur la nécessité de la responsabilité sociale et des politiques publiques pour assurer la sécurité alimentaire et nutritionnelle des familles qui utilisent ce programme social.

Mots-clés: securite alimentaire et nutritionnelle; la pauvrete; évaluation de la sante; santé publique; politique nutritionnelle.

Resumen: Este estudio analiza el Bolsa Familia en los aspectos de seguridad alimentaria y nutricional de beneficiarios de la ciudad de Vitória da Conquista, BA. De los formularios de solicitud semiestructurados con los beneficiarios y las observaciones de campo, el diagnóstico mostró la necesidad de responsabilidad social y políticas públicas para asegurar la seguridad alimentaria y nutricional de las familias que utilizan este programa social.

Palabras clave: seguridad alimentaria y nutricional; pobreza; evaluación de la salud; salud pública; política nutricional.

\footnotetext{
${ }^{1}$ Universidade Federal do Vale do São Francisco (UNIVASF), Petrolina, Pernambuco, Brasil.

2 Escola Nacional de Saúde Pública Sergio Arouca (ENSP), Fundação Oswaldo Cruz, Rio de Janeiro, Brasil.
} 


\section{INTRODUÇÃO}

Os programas de transferência de renda têm se colocado no cenário público contemporâneo como um dos instrumentos de redução da desigualdade e de alívio imediato da pobreza, inicialmente em âmbito municipal e, depois, nacional de diversos programas de transferência de renda, que associam a manutenção do pagamento do benefício ao cumprimento de contrapartidas pela família nas áreas de educação e saúde (BELIK, 2003).

Os estudos sobre segurança alimentar devem considerar nas suas análises grupos populacionais vulneráveis que, por sua condição biológica, são mais suscetíveis à ingestão deficitária de alimentos. Existem cinco métodos comumente utilizados para mensurar a segurança alimentar (PEREIRA; SANTOS, 2008). Nesse contexto, no desenvolvimento de uma vigilância alimentar e nutricional no combate à fome e à pobreza, insere-se a abordagem das políticas públicas voltadas para a assistência social, promoção da saúde e da Segurança Alimentar e Nutricional (SAN) ${ }^{3}$, interferindo positivamente nas condições de vida da população mais carente.

O Programa Bolsa Família (PBF) ${ }^{4}$ visa amortizar a pobreza, as desigualdades sociais e combater a fome entre as famílias pobres vitimadas pela desigualdade social (SOARES; SÁTYRO, 2009). Assim, o PBF foi constituído com o objetivo de pôr em prática uma nova concepção de assistência social, ou seja, de suprir as necessidades básicas de uma população-alvo com requisitos predefinidos através de um recebimento (benefício) e articulado com o desenvolvimento de capacidades para maior autonomia social. Foi instituído pela Medida Provisória n. 132, de 20 de outubro de 2003, posteriormente convertida na Lei n. 10.836 (BRASIL, 2011). Busca uma transferência direta de renda com condicionalidades destinada às famílias que atendam aos critérios de elegibilidade preconizados pelo programa, quais sejam, o acompanhamento em saúde (exame pré-natal, por exemplo), nutricional e vacinação, a manutenção dos filhos na escola com frequência escolar acima de determinado patamar (VAITSMAN; ANDRADE; FARIAS, 2009). Com efeito, o PBF, fundamentou-se na previsibilidade da melhoria e renda e dos padrões de alimentação, as quais, em consonância com ações de saúde e nutrição, integram o conjunto de condições a serem desempenhadas pelas famílias (PANIGASSI et al., 2008).

A política de saúde compõe o conjunto de ações governamentais relacionado à proteção social brasileira integrando um dos fundamentos do Estado: a Seguridade Social, aprovada desde 1988 dentro da Constituição Federal. Posteriormente, em 1990, a saúde passou a ser regula-

\footnotetext{
${ }^{3}$ A Segurança Alimentar e Nutricional (SAN) é definida como "a realização do direito de todos ao acesso regular e permanente a alimentos de qualidade e em quantidade suficiente, sem comprometer o acesso a outras necessidades essenciais, tendo como base práticas alimentares promotoras de saúde, que respeitem a diversidade cultural e que sejam social, econômica e ambientalmente sustentáveis" (BRASIL, 2006).

Foi criada pela lei 11.346 (BRASIL, 2006), a qual versa que todo brasileiro tem direito a uma alimentação saudável, acessível, com qualidade, em quantidade suficiente, de forma estável, baseada em práticas alimentares promotoras de saúde, sem comprometer outras necessidades básicas, respeitando as singularidades culturais de cada região, sendo social e economicamente sustentáveis. Com efeito, o conceito SAN foi, pela primeira vez, colocado em sua plenitude, ligado não mais a um salário limite entre a dignidade e a pobreza, mas a uma situação de desenvolvimento integral do ser humano. A SAN só existe se todas as necessidades básicas do ser humano forem acatadas simultaneamente, proporcionando aos indivíduos capacidade de gerência e reflexão sobre suas próprias condições de vida. Logo, a satisfação das necessidades humanas básicas sinaliza a ideia de existência de um conjunto de satisfações indivisíveis e concomitantes; ou seja, se um ou todos fracassam, o risco para o prosseguimento da vida é o mesmo e é grave (PESSANHA, 2004).

${ }^{4}$ O Programa Bolsa Família (PBF) constitui um dos maiores bancos de dados municipais sobre o perfil social da população atendida auxiliando a realização de ações conjuntas, as quais incluem atividades que promovem geração de renda, educação nutricional e capacitação dos beneficiários para o mercado de trabalho (SOARES; SÁTYRO, 2009).
} 
mentada pela Lei 8.080, que dispõe sobre a Lei Orgânica de Saúde e, em 1996, sendo regida pela Norma Operacional Básica do Sistema Único de Saúde (NOB-SUS) (BRASIL, 1988). A Lei Orgânica de Saúde, em seu art. 2ํㅡㄹ diz: "A saúde é um direito fundamental do ser humano, devendo o Estado prover as condições indispensáveis ao seu pleno exercício; § 1 으 O dever do Estado em garantir a saúde consiste na formulação e execução de políticas econômicas e sociais que visem à redução de riscos de doenças e de outros agravos e no estabelecimento de condições que assegurem acesso universal e igualitário às ações e aos serviços para sua promoção, proteção e recuperação" (BRASIL, 1990).

A dificuldade de acesso regular e permanente aos alimentos por um contingente significativo da população brasileira, associada à renda insuficiente, determina um quadro de insegurança alimentar (IA) (PEREIRA; SANTOS, 2008). Por conseguinte, quanto menor o rendimento mensal domiciliar per capita, maior a proporção de domicílios em situação de IA moderada ou grave, fato esse que revela a restrição quantitativa e qualitativa de alimentos, incorrendo, consequentemente, em fome (COTTA; MACHADO, 2013). Os números da Pesquisa Nacional por Amostra de Domicílios (PNAD), realizada pelo Instituto Brasileiro de Geografia e Estatística (IBGE) em 2014, revelam que a IA atinge 52 milhões, sendo o Nordeste a região mais afetada por esse problema (46,1\%), enquanto o número não passa de 18,7\% (BRASIL, 2014).

Os estudos de impacto do PBF na SAN observaram que, prioritariamente, a renda transferida é investida em alimentos e, dessa forma, determina mudanças no padrão alimentar das famílias (MELGAR-QUIÑONES; HACKETT, 2008; PEREIRA; SANTOS, 2008; SEGALL-CORRÊA; LEON-MARIN, 2009). Uma análise feita baseada em amostra nacional representativa das famílias inscritas no PBF mostrou um aumento de $79 \%$ na quantidade de alimentos adquiridos por aquelas famílias que estavam sofrendo de IA grave no início da pesquisa, em comparação com aumento de $60 \%$ entre as famílias que relataram segurança alimentar (PANIGASSI et al., 2008).

Pesquisa realizada pela Faculdade de Saúde Pública da Universidade de São Paulo (USP) associou o programa de distribuição de renda do Governo Federal à melhora na qualidade e quantidade de alimentos consumidos pelos beneficiários. Segundo o estudo, que se baseou nos dados da Pesquisa de Orçamento Familiar (POF), realizada pelo IBGE em 2008 e 2009, o PBF permitiu às famílias beneficiadas aproximadamente mais 100 kcal diárias por pessoa (SANTOS; PASQUIM; SANTOS, 2011). Também foi observado um aumento maior na variedade de alimentos entre famílias em situação de IA grave $(68,7 \%)$ e moderada $(77,6 \%)$ do que entre aquelas em Segurança Alimentar (59,6\%); concluindo que, mesmo com a percepção de aumento na quantidade e na variedade dos alimentos, a partir do recebimento do benefício do PBF, a situação de IA é alta, uma vez que, apesar do aumento declarado no consumo de alimentos, uma parcela significativa dos(as) beneficiários(as) (21\%, representando 2,3 milhões de famílias), encontram-se em situação de IA grave (fome entre adultos e/ou crianças da família); outros 34\% (ou 3,8 miIhões de famílias) estão em situação de IA moderada (restrição na quantidade de alimentos na família). Apresentam IA leve, em que não há falta de alimentos, mas preocupação em relação ao consumo no futuro, $28 \%$ (ou 3,1 milhões de famílias), e 17\% (ou 1,9 milhão de famílias) estão em situação de segurança alimentar e nutricional.

Nesse contexto, esta pesquisa buscou avaliar os impactos do PBF, tendo como base analítica os elementos de SAN relativa aos beneficiários no município de Vitória da Conquista, BA. A partir disso, espera-se fornecer subsídios importantes para uma reflexão mais aprofundada sobre o funcionamento do programa ao nível local e repercussões. 


\section{MÉTODO}

Trata-se de um estudo transversal analítico, realizado com famílias beneficiárias do PBF de Vitória da Conquista, BA, no período de setembro a dezembro de 2015.

Para a avaliação das características dos domicílios, foram selecionadas as seguintes variáveis: (a) condições de saneamento básico (tratamento do esgoto, coleta de lixo, abastecimento de água e tratamento doméstico da água); (b) renda familiar mensal per capita (total de rendimentos da família dividido pelo número de moradores, expressa em múltiplos do salário mínimo); (c) número de pessoas por domicílio; (d) escolaridade do chefe da família (categorizada em: analfabeto, ensino fundamental incompleto, ensino fundamental completo, ensino médio completo e curso universitário completo); e (e) classificação socioeconômica pelo critério da Associação Brasileira de Institutos de Pesquisa de Mercados (ABIPEME), que considera a presença de bens e serviços do domicílio e a escolaridade do chefe da família (MATTAR, 1995).

De acordo com o Ministério do Desenvolvimento Social e Combate à Fome (BRASIL, 2008 ), existiam em outubro de 2014, 28774 famílias beneficiadas pelo PBF em Vitória da Conquista. Calculando estatisticamente o tamanho da amostra, com um erro amostral de $5 \%$ e nível de confiança de 95\% (SILVA, 2001), chegou-se à conclusão de que, para a amostra ser representativa, seria necessária a aplicação de 230 entrevistas.

Os dados foram coletados nas 10 Unidades de Saúde da Família (USF), sendo cinco localizadas na zona urbana (USF Nossa Senhora Aparecida, USF Conveima, USF Nelson Barros, USF Patagônia e USF Recanto das Águas) e cinco na zona rural (USF Lagoa das Flores, USF Pradoso, USF Bate Pé, USF Limeira e USF José Gonçalves). A escolha das unidades se deu por conveniência, buscando a otimização da coleta de dados de pesquisa, o que não interferiu nos resultados.

Antes de iniciar a entrevista, foi lido o Termo de Consentimento Livre e Esclarecido (TCLE) para o entrevistado em um local que garantia a privacidade. Foram utilizados questionários estruturados e validados, adaptados de Anschau, Matsuo e Segall-Corrêa (2012), por meio dos quais foram coletadas informações Sociais, Econômicas e Ambientais dos beneficiários. O método para a avaliação da segurança alimentar na população brasileira é denominado Escala Brasileira de Insegurança Alimentar (EBIA $)^{5}$, validada para o Brasil por Segall-Corrêa et al. (2003), e tem sido reconhecida como indicador sensível para detectar o risco de insegurança alimentar.

As associações entre as variáveis independentes com a variável dependente foram estabelecidas utilizando-se os testes de Qui-quadrado e razão de chances com o respectivo intervalo de confiança de 95\% para as variáveis categóricas. Foi adotado o nível de significância de 5\%. A análise estatística foi realizada no programa SAS/STAT versão 8.2.

Este estudo recebeu aprovação do Comitê de Ética em Pesquisa com Seres Humanos (46081015.7.0000.5240) (Escola Nacional de Saúde Pública Sergio Arouca/Fundação Oswaldo Cruz).

\footnotetext{
${ }^{5}$ A aplicação da EBIA apresenta uma vantagem em seu uso, pois mede o fenômeno diretamente a partir da experiência de insegurança alimentar (IA) vivenciada e percebida pelas pessoas afetadas, captando não só a dificuldade de acesso aos alimentos, mas também a dimensão psicossocial da IA (CORRÊA, 2007). Constitui-se em quatro categorias: (a) segurança alimentar; (b) insegurança alimentar leve (IA leve); (c) insegurança alimentar moderada (IA moderada) e (d) insegurança alimentar grave (IA grave). Essa escala é elaborada a partir de questionário com 15 perguntas referentes à experiência nos últimos três meses de insuficiência alimentar em diversos níveis de intensidade. Inclui desde a preocupação de que a comida possa acabar até a vivência de passar um dia todo sem comer. Cada resposta afirmativa do questionário corresponde a um ponto, portanto, a soma de todas as repostas varia de 0 a 15 pontos.
} 


\section{RESULTADOS E DISCUSSÃO}

\subsection{Perfil dos beneficiários}

Dos 230 beneficiários entrevistados, 97,1\% ( $n=173)$ residiam na zona urbana e $24,8 \%$ $(\mathrm{n}=57)$ na zona rural do município de Vitória da Conquista, BA. Em relação à água de consumo, $81,7 \%$ ( $n=188$ ) informaram que era proveniente da rede pública de abastecimento. Quanto ao esgotamento sanitário, 60\% ( $n=138)$ informaram que a cobertura de coleta sanitária era da rede pública. A coleta de lixo domiciliar municipal atendia a 75,2\% $(n=173)$ das residências.

O padrão sanitário dos domicílios que integraram a pesquisa foi satisfatório, pois foi verificado água de consumo de boa qualidade e elevada disponibilidade, presença de fossa séptica ou rede pública para esgotamento, com regular coleta domiciliar de lixo. Esses dados se assemelham aos encontrados por Motta et al. (2004) em estudo realizado em Piracicaba/SP, onde foram descritas boas condições de saneamento básico, com sistema de esgotamento adequado em todas as residências e água disponível em domicílios de baixa renda.

Na constituição familiar dos beneficiários, foi predominante o número de 4 a 6 moradores $(59,6 \%, n=137)$ e a maior parte dos domicílios $(88,7 \%, n=204)$ apresentou algum morador menor de 18 anos. Segundo a estratificação de renda, em 42,1\% dos domicílios a renda per capita foi de $R \$ 77,00$ ou menos, enquanto que em $27,8 \%$ a renda foi maior que meio salário mínimo. Chama atenção o fato de que, em $72,2 \%$, a renda per capita apresentou valores inferiores a $1 / 2$ salário mínimo brasileiro. O valor do benefício recebido por 31,3\% dos beneficiários foi superior a $\mathrm{R} \$ 189,00$ (Tabela 1).

Tabela 1 - Composição das 230 famílias pesquisadas, Vitória da Conquista, BA, 2015

\begin{tabular}{lccccc}
\hline \multirow{2}{*}{ Moradores } & \multicolumn{2}{c}{ Frequência } & \multicolumn{2}{c}{ IC 95\% } \\
\cline { 5 - 6 } \cline { 4 - 5 } & N & \% & Limite Inferior (\%) & Limite Superior (\%) \\
\hline Número de Moradores & & & & \\
3 moradores ou menos & $64 / 230$ & 27,8 & 22,4 & 33,9 \\
4 a 6 moradores & $137 / 230$ & 59,6 & 53,1 & 65,7 \\
7 moradores ou mais & $29 / 230$ & 12,6 & 8,9 & 17,5 \\
Menores de 18 anos & & & & 92,1 \\
Sim & $204 / 230$ & 88,7 & 83,9 & 16 \\
Não & $26 / 230$ & 11,3 & 7,8 & \\
\hline
\end{tabular}

IC: intervalo de confiança

Fonte: Suzart (2016).

Considerando dados de classificação econômica, 75,1\% (n=173) das famílias entrevistadas se enquadram nas classes $D$ ou $E$, das quais, $67,3 \%(n=155)$ na classe $D$ e $7,8 \%(n=18)$ na classe $E$, indicativo de um grande número de famílias com baixa classificação econômica. O chefe do domicílio na maioria das famílias era do sexo masculino 64,7\% ( $n=149)$. O grau de escolaridade obtido de $40,4 \%$ ( $n=93$ ) foi analfabeto ou primário incompleto e somente $21,7 \%$ possuem entre a 5a a 8a série completa ou segundo grau incompleto ou completo. O vínculo empregatício de $45,2 \%(n=104)$ é representado pelo trabalho formal; entretanto, 31,3\% ( $n=72)$ trabalham na informalidade e 16,5\% (n=38) estão desempregados (Tabela 2). 
Tabela 2 - Classificação econômica das 230 famílias pesquisadas, Vitória da Conquista, BA, 2015

\begin{tabular}{|c|c|c|c|c|}
\hline \multirow{2}{*}{ Variáveis } & \multicolumn{2}{|c|}{ Frequência } & \multicolumn{2}{|c|}{ IC 95\% } \\
\hline & $\mathbf{N}$ & $\%$ & Limite inferior (\%) & Limite superior (\%) \\
\hline \multicolumn{5}{|l|}{ Renda per capita } \\
\hline $\mathrm{R} \$ 77,00$ ou menos & $97 / 230$ & 42,1 & 35,9 & 48,6 \\
\hline $\mathrm{R} \$ 77,01$ a $\mathrm{R} \$ 154,00$ & $42 / 230$ & 18,2 & 13,8 & 23,7 \\
\hline $\mathrm{R} \$ 154,01$ a $1 / 2 \mathrm{SM}$ & $27 / 230$ & 11,7 & 8,1 & 16,5 \\
\hline Maior $1 / 2$ SM & $64 / 230$ & 27,8 & 22,4 & 33,9 \\
\hline \multicolumn{5}{|l|}{ Benefício recebido } \\
\hline $\mathrm{R} \$ 77,00$ ou menos & $62 / 230$ & 26,9 & 21,6 & 33 \\
\hline $\mathrm{R} \$ 77,01$ a $\mathrm{R} \$ 112,00$ & $49 / 230$ & 21,3 & 16,5 & 27 \\
\hline $\mathrm{R} \$ 112,01$ a $\mathrm{R} \$ 189,00$ & $47 / 230$ & 20,4 & 15,7 & 26,1 \\
\hline Maior $\mathrm{R} \$ 189,00$ & $72 / 230$ & 31,3 & 25,6 & 37,5 \\
\hline \multicolumn{5}{|l|}{ Classificação Econômica } \\
\hline Classe C & $57 / 230$ & 24,7 & 19,6 & 30,7 \\
\hline Classe D & $155 / 230$ & 67,3 & 61 & 73,1 \\
\hline Classe E & $18 / 230$ & 7,8 & 5 & 12 \\
\hline
\end{tabular}

IC: intervalo de confiança

Fonte: Suzart (2016).

\subsection{Insegurança alimentar e Bolsa Família}

A política de segurança alimentar e nutricional possui, na sua concepção, um caráter multidimensional e intersetorial, devendo, portanto, na sua gestão, estar interligada aos diferentes níveis de governo e também à sociedade civil, o que lhe proporcionará um maior alcance e efetividade (TRALDI et al., 2012; RABELO; RUCKERT, 2014). Tais aspectos estão contemplados nesse marco regulatório, em especial, no Sistema Nacional de Segurança Alimentar (SISAN). Contudo a adesão dos estados e municípios ao SISAN ainda está em andamento.

No Brasil, em 2004, 34\% dos domicílios foram classificados como tendo algum tipo ou grau de restrição alimentar, atingindo cerca de 72 milhões de pessoas. A prevalência de domicílios com IA aumentava para 66\%, considerando aqueles onde residia algum beneficiário de programas de transferência de renda (SUZART, 2016). A Tabela 3 explicita que a prevalência de IA foi de $73,5 \%$ ( $n=169)$, distribuídas em IA leve $(40,8 \%, n=94)$, moderada $(26,9 \%, n=62)$ ou severa $(5,6 \%, n=13)$.

Tabela 3 - Insegurança alimentar das famílias beneficiárias, Vitória da Conquista, BA, 2015

\begin{tabular}{lccccc}
\hline \multirow{2}{*}{ Nível de insegurança alimentar } & \multicolumn{2}{c}{ Prevalência } & \multicolumn{2}{c}{ IC 95\% } \\
\cline { 2 - 5 } & $\mathbf{N}$ & $\mathbf{\%}$ & Limite inferior (\%) & Limite superior (\%) \\
\hline Segurança alimentar & 61 & 26,6 & 21,2 & 32,5 \\
IA leve & 94 & 40,8 & 34,7 & 47,3 \\
IA moderada & 62 & 26,9 & 21,6 & 33 \\
IA severa & 13 & 5,7 & 3,3 & 9,4 \\
\hline Total & $\mathbf{2 3 0}$ & $\mathbf{1 0 0}$ & & \\
\hline
\end{tabular}

IA: insegurança alimentar; IC: intervalo de confiança

Fonte: Suzart (2016). 
$\mathrm{Na}$ Tabela 4, verifica-se que a IA leve foi a mais prevalente em 2 das 4 categorias de distribuição de renda per capita, porém não existindo uma associação significativa $(p>0,05)$ entre os níveis de (in)segurança alimentar e a renda familiar per capita. A prevalência de IA moderada ou severa na categoria de renda per capita de $\mathrm{R} \$ 77,00$ ou menos $(55,7 \%)$ foi cerca de dezesseis vezes superior à observada entre os indivíduos com renda de $\mathrm{R} \$ \frac{1}{2} 2$ salário mínimo ou mais $(3,4 \%)$.

Ficou evidente que, conforme se reduz a renda per capita, as chances de a família vir a apresentar IA moderada ou severa aumentavam progressivamente. A razão de chances mais elevada para essa condição, OR 35,16 (IC 4,59-268,93), ocorre nos domicílios com moradores que apresentam renda per capita de $\mathrm{R} \$ 77,00$ ou menos. A classificação econômica não mostrou associação significativa com a IA. Porém, ao se comparar as classes D ou E com a C, mostra que a razão de chances para IA moderada ou severa foi maior entre as famílias de classificação econômica mais baixa $(O R=1,53$ - IC $0,78-2,98)$, indicando que essas famílias estão mais propensas à experiência da fome, entre adultos ou moradores menores de 18 anos.

Tabela 4 - Insegurança alimentar segundo caracterização econômica das famílias beneficiárias, Vitória da Conquista, BA, 2015

\begin{tabular}{|c|c|c|c|c|c|c|c|c|}
\hline \multirow{3}{*}{$\begin{array}{c}\text { Dados econômicos } \\
\text { Renda familiar per capita }\end{array}$} & \multicolumn{8}{|c|}{ Nível de (in)segurança alimentar } \\
\hline & \multicolumn{2}{|c|}{ SA } & \multicolumn{2}{|c|}{ IA leve } & \multicolumn{2}{|c|}{$\begin{array}{c}\text { IA moderada/ } \\
\text { severa }\end{array}$} & \multirow[t]{2}{*}{$\mathrm{OR}^{*}$} & \multirow[t]{2}{*}{ IC 95\% } \\
\hline & $\mathbf{N}$ & $\%$ & $\mathbf{N}$ & $\%$ & $\mathbf{N}$ & $\%$ & & \\
\hline $\mathrm{R} \$ 77,00$ ou menos & 6 & 6,2 & 37 & 38,1 & 54 & 55,7 & 35,16 & $4,59-268,93$ \\
\hline$R \$ 77,01$ a $R \$ 154,00$ & 11 & 26,2 & 18 & 42,8 & 13 & 31 & 12,55 & $1,53-102,41$ \\
\hline $\mathrm{R} \$ 154,01$ a $1 / 2 \mathrm{SM}$ & 24 & 38,7 & 31 & 50 & 7 & 11,3 & 3,56 & $0,41-30,41$ \\
\hline Maior $1 / 2$ SM & 20 & 69 & 8 & 27,6 & 1 & 3,4 & 1 & Referência \\
\hline \multicolumn{9}{|l|}{ Classificação Econômica } \\
\hline Classe C & 18 & 31 & 25 & 43,1 & 15 & 25,8 & 1 & Referência \\
\hline Classe D ou E & 43 & 25 & 69 & 40,2 & 60 & 34,8 & 1,53 & $0,78-2,98$ \\
\hline
\end{tabular}

Teste Qui-quadrado, valor de $p>0,05$ para as duas variáveis; *Odds Ratio bruto; SM: salário mínimo; SA: segurança alimentar; IC: intervalo de confiança; IA: insegurança alimentar

Fonte: Suzart (2016).

Foi observado que nos domicílios onde há presença de moradores menores de 18 anos a IA leve $(41,6 \%, n=85)$ e a IA moderada ou severa $(32,5 \%, n=204)$ estão mais presentes quando comparadas aos domicílios sem moradores nesta faixa etária. Importante destacar que nas famílias onde não há a presença de moradores com menos de 18 anos, a prevalência de Segurança Alimentar $(30,8 \%)$ foi um pouco superior à apresentada nas famílias com membros menores de 18 anos $(25,9 \%)$ e que a IA moderada ou severa (34,6\%) não apresentam diferenças comparativas às residências com moradores menores de 18 anos. Porém uma vez que o valor de $p=0,8721$ não se pode afirmar a existência de uma relação entre estes dados.

A Tabela 5 apresenta os dados referentes a IA segundo o número de membros no domicilio onde esta variável não interfere nos níveis de IA $(p=0,1266)$. Porém, nos domicílios com sete moradores ou mais, a IA moderada ou severa apresenta valores superiores quando comparadas com famílias com 3 moradores ou menos, mostrando que, mesmo não havendo relação entre as variáveis, os resultados sugerem tendência de aumento da IA moderada ou severa com o aumento do número de membros do domićlio. 
Tabela 5 - Insegurança alimentar segundo o número de membros no domicílio das famílias beneficiárias, Vitória da Conquista, BA, 2015

\begin{tabular}{|c|c|c|c|c|c|c|c|c|}
\hline \multirow{3}{*}{ N. de membros domicílio } & \multicolumn{6}{|c|}{ Nível de (in)segurança alimentar } & \multirow{2}{*}{\multicolumn{2}{|c|}{ Total }} \\
\hline & \multicolumn{2}{|c|}{ SA } & \multicolumn{2}{|c|}{ IA leve } & \multicolumn{2}{|c|}{$\begin{array}{c}\text { IA moderada/ } \\
\text { severa }\end{array}$} & & \\
\hline & $\mathbf{N}$ & $\%$ & $\mathbf{N}$ & $\%$ & $\mathbf{N}$ & $\%$ & $\mathbf{N}$ & $\%$ \\
\hline 3 ou menos & 18 & 28,1 & 26 & 40,6 & 20 & 31,3 & 64 & 27,83 \\
\hline 4 a 6 & 37 & 27 & 61 & 44,5 & 39 & 28,5 & 137 & 59,56 \\
\hline 7 ou mais & 6 & 20,7 & 7 & 24,1 & 16 & 55,2 & 29 & 12,61 \\
\hline Total & 61 & 26,5 & 94 & 40,9 & 75 & 32,6 & 230 & 100 \\
\hline
\end{tabular}

Teste Qui-quadrado, valor de $p=0,1266$; SA: segurança alimentar; IA: insegurança alimentar Fonte: Suzart (2016).

Entre as variáveis relacionadas ao chefe do domicílio, nenhuma delas apresentou diferença significativa (significância de 5\%), sendo as mais próximas de apresentarem uma relação significativa o sexo $(p=0,0599)$ e a faixa etária $(p=0,0885)$ (Tabela 6). Observou-se que a IA moderada ou severa foi predominante em domicílios com chefe da família do sexo feminino (42\%) e que embora a faixa etária não tenha sido associada à IA, notou-se uma tendência no aumento da IA moderada ou severa com o aumento da idade do chefe do domicílio. A escolaridade e o vínculo empregatício do chefe do domicílio não interferiram de forma significativa na IA em seus níveis, sendo o valor de $p$ igual a 0,0599 e 0,0979, respectivamente. Porém pode-se verificar que quanto maior a escolaridade, mais próxima a família se encontra em relação à segurança alimentar.

Tabela 6 - Insegurança alimentar segundo dados do chefe do domicílio das famílias beneficiárias, Vitória da Conquista, BA, 2015

\begin{tabular}{|c|c|c|c|c|c|c|c|c|c|}
\hline \multirow{3}{*}{ Variável } & \multicolumn{6}{|c|}{ Nível de (in)segurança alimentar } & \multirow{2}{*}{\multicolumn{2}{|c|}{ Total }} & \multirow{3}{*}{ Valor de $p$} \\
\hline & \multicolumn{2}{|c|}{ SA } & \multicolumn{2}{|c|}{ IA leve } & \multicolumn{2}{|c|}{$\begin{array}{c}\text { IA moderada/ } \\
\text { severa }\end{array}$} & & & \\
\hline & $\mathbf{N}$ & $\%$ & $\mathbf{N}$ & $\%$ & $\mathbf{N}$ & $\%$ & $\mathbf{N}$ & $\%$ & \\
\hline \multicolumn{10}{|l|}{ Sexo } \\
\hline Masculino & 38 & 25,5 & 70 & 47 & 41 & 27,5 & 149 & 64,78 & \multirow{2}{*}{0,0599} \\
\hline Feminino & 23 & 28,4 & 24 & 29,6 & 34 & 42 & 81 & 35,22 & \\
\hline \multicolumn{10}{|l|}{ Escolaridade } \\
\hline 1 & 22 & 23,6 & 39 & 42 & 32 & 34,4 & 93 & 40,43 & \multirow{3}{*}{0,0599} \\
\hline 2 & 20 & 23 & 36 & 41,4 & 31 & 35,6 & 87 & 37,83 & \\
\hline 3 & 19 & 38 & 19 & 38 & 12 & 24 & 50 & 21,74 & \\
\hline \multicolumn{10}{|l|}{ Vínculo empregatício } \\
\hline Formal & 35 & 33,6 & 32 & 30,8 & 37 & 35,6 & 104 & 45,22 & \multirow{4}{*}{0,0979} \\
\hline Informal & 17 & 23,6 & 33 & 45,8 & 22 & 30,6 & 72 & 31,3 & \\
\hline Não trabalha & 4 & 10,5 & 22 & 57,9 & 12 & 31,6 & 38 & 16,52 & \\
\hline Aposentado & 5 & 31,3 & 7 & 43,7 & 4 & 25 & 16 & 7,16 & \\
\hline \multicolumn{10}{|l|}{ Faixa etária } \\
\hline 29 ou menos & 18 & 38,3 & 20 & 42,5 & 9 & 19,2 & 47 & 20,43 & \multirow{3}{*}{0,0885} \\
\hline 30 a 49 anos & 31 & 23,5 & 57 & 43,9 & 44 & 33,4 & 132 & 57,39 & \\
\hline 50 anos ou mais & 12 & 23,5 & 17 & 33,4 & 22 & 43,1 & 51 & 22,18 & \\
\hline
\end{tabular}

Teste Qui-quadrado, valor de p; SA: segurança alimentar; IA: insegurança alimentar Fonte: Suzart (2016). 
Famílias que vivem em situação de IA têm dificuldades no consumo de certos grupos de alimentos. Perez-Escamilla et al. (2004) descrevem menor probabilidade do consumo diário de carnes, derivados do leite, frutas e verduras, feijão. Por isso a insuficiência alimentar pode levar à carência de macro e micronutrientes (SEGALL-CORRÊA; LEON-MARIN, 2009).

Mesmo a variável renda per capita não tendo apresentado associação significativa com a IA, pode-se observar uma relação entre estas variáveis. Foi observado que a prevalência de IA moderada ou severa na categoria de menor renda per capita foi superior à observada entre os indivíduos com maior renda, mostrando que, em famílias com renda per capita mais baixa, não permite aos seus membros condições favoráveis de alimentação, o que corrobora com diversos estudos, nacionais e internacionais, que registraram a baixa renda como fato determinante mais importante da IA e fome (SEGALL-CORRÊA; LEON-MARIN, 2009; ANSCHAU; MATSUO; SEGALL-CORRÊA, 2012).

Dados deste estudo mostram uma maior prevalência de IA moderada ou severa se comparada com a segurança alimentar, porém, o fato de essa última condição também ser observada, destacando quando se analisou a relação entre IA e renda per capita, mostra que algumas famílias, mesmo em extrema pobreza, conseguiram se manter em segurança alimentar. Tal fato demonstra que nem todas as pessoas ficam passivas diante da IA coincidindo com estudo realizado por Maxwell (1995), no qual se evidencia que muitas famílias conseguem elaborar estratégias na tentativa de superação desta condição.

A evolução histórica da política social brasileira em direção à construção de uma política pública de transferência de renda, orientada para a focalização em grupos considerados vulneráveis, consolida-se como mecanismo central na composição do sistema brasileiro de proteção social. A proteção social no Brasil está inserida na concepção de seguridade social, isto é, no conjunto de seguranças sociais que uma sociedade, de forma solidária, garante a seus membros. Portanto a centralidade está no processo histórico de cada sociedade e, nele, o trânsito pelo qual determinadas condições sociais de dignidade e sobrevivência são asseguradas enquanto um direito social universal.

\section{CONCLUSÕES}

Evidencia a pesquisa em seu contexto maior que o PBF alcança alguns produtos, tais como aumento da frequência escolar e cumprimento da agenda de acompanhamento da saúde. No entanto a transferência de renda por si só não é suficiente para a superação da pobreza e das desigualdades. Urge que seja integrada a transferência de renda a estratégias mais amplas, articulando desenvolvimento econômico e desenvolvimento social, e assim, contemplando a pobreza em seu caráter multidimensional.

Entretanto cabe destacar que o PBF representa a conquista de segmentos populares da sociedade brasileira que lutam por melhores expectativas e condições de vida, ainda que isso implique a concessão desses setores permitindo o domínio dos setores elitistas, que, ao concederem esses benefícios às classes populares, consolidam sua hegemonia social, política e econômica. Diante disso, mesmo apresentando inúmeras limitações (baixo valor auferido, abrangência insuficiente em relação ao conjunto de potenciais beneficiários, precariedade e/ ou de programas complementares, não constitui um direito adquirido etc.), o Programa tem repercutido de forma positiva nas vidas cotidianas dos beneficiários que vivem em situações de 
miséria e pobreza, sobretudo por ser, em muitos casos, a única renda familiar segura, chegando a representar o principal meio de manutenção da vida.

\section{REFERÊNCIAS}

ANSCHAU, F. R.; MATSUO, T.; SEGALL-CORRÊA, A. M. Insegurança alimentar entre beneficiários de programas de transferência de renda. Revista de Nutrição, Campinas, SP, v. 25, n. 2, p. 177-89, mar./abr. 2012.

BELIK, W. Perspectivas para segurança alimentar e nutricional no Brasil. Saúde e Sociedade, São Paulo, v. 12, n. 1, p. 12-20, jan./jun. 2003.

BRASIL. Senado Federal. Pesquisa Nacional por Amostra de Domicílios. Insegurança Alimentar. 2014. Disponível em: <http://agenciabrasil.ebc.com.br/geral/noticia/2014-12/IBGE-52-milhoesde-brasileiros-em-situa\%C3\%A7ao-de-inseguranca-alimentar>. Acesso em: 25 jan. 2016.

. Ministério do Desenvolvimento Social e Combate à Fome (MDS). 2008. Disponível em: <http://www.mds.gov.br/webarquivos/legislacao/bolsa_familia/_doc/portarias/2008/ Portaria_GM_MDS_220_27-06-08-1.pdf>. Acesso em: 18 maio 2016.

Lei n. 11.346, de 15 de setembro de 2006. Lei Orgânica de Segurança Alimentar e Nutricional. Brasília, DF, 2006. Lei Orgânica de Saúde n. 8.080, de 19 de setembro de 1990. Brasília, DF, 1990. Secretaria Especial de Editoração e Publicações. Constituição da República Federativa do Brasil. Brasília, DF, 1988.

Pesquisa Nacional por Amostra de Domicílios. Aspectos complementares de educação e acesso a transferências de renda de Programas sociais. Disponível em: <http://www. ibge.gov.br/home/estatistica/pesquisas/pesquisa_resultados.php?id_pesquisa=40>. Acesso em: 31 jan. 2016.

CORRÊA, A. M. S. Insegurança alimentar medida a partir da percepção das pessoas. Estudos Avançados, São Paulo, v. 21, n. 60, p. 143-54, maio/ago. 2007.

COTTA, R. M. M.; MACHADO, J. C. Programa Bolsa Família e segurança alimentar e nutricional no Brasil: revisão crítica da literatura. Revista Panamericana de Salud Pública, v. 33, n. 1, p. 5460, 2013.

MATTAR, F. N. Análise crítica dos estudos de estratificação socioeconômica de ABA-ABIPEME. RAE - Revista de Administração de Empresas, São Paulo, v. 30, n. 1, p. 57-74, jan./mar. 1995.

MAXWELL, D. G. Measuring food insecurity: the frequency and severity of "coping strategies". Washington: Food Consumption and Nutrition Division (FCND), 1995. (Discussion paper n. 8).

MOTTA, D. G. et al. Consumo alimentar de famílias de baixa renda no município de Piracicaba/ SP. Saúde em Revista, Piracicaba, SP, v. 6, n. 13, p. 63-70, 2004.

PANIGASSI, G. et al. Insegurança alimentar como indicador de iniquidade: análise de inquérito populacional. Cadernos de Saúde Pública, Rio de Janeiro, v. 24, n. 10, p. 2376-84, out. 2008.

PEREIRA, R. A.; SANTOS, L. M. P. A dimensão da insegurança alimentar. Revista de Nutrição, Campinas, SP, v. 21 (suplemento), p. 7-13, jul./ago. 2008.

PÉREZ-ESCAMILLA, R. et al. An adapted version of the U.S. Department of Agriculture Food Insecurity module is a valid tool for assessing household food insecurity in Campinas, Brazil. The Journal of Nutrition, v. 134, n. 8, p. 1923-8, ago. 2004. 
PESSANHA, L. D. R. A experiência brasileira em políticas públicas para a garantia do direito ao alimento - breve histórico. Cadernos de Debates, Campinas, SP, n. 11, p. 1-37, dez. 2004.

RABELO, M. M.; RUCKERT, N. A construção da política de segurança alimentar e nutricional: o caso de Porto Alegre. Indicadores Econômicos FEE, Porto Alegre, RS, v. 41, n. 3, p. 85-100, 2014.

SANTOS, L. M. P.; PASQUIM, E. M.; SANTOS, S. M. C. Programas de transferência de renda no Brasil: um estudo multidimensional da implementação do Bolsa Escola, Bolsa Alimentação e Cartão Alimentação. Revista Ciência \& Saúde Coletiva, Rio de Janeiro, v. 16, n. 3, p. 1821-34, 2011.

SEGALL-CORRÊA, A. M.; LEON-MARIN, L. A Segurança alimentar no Brasil: proposição e usos da Escala Brasileira de Medida da Insegurança Alimentar (EBIA) de 2003 a 2009. Segurança Alimentar e Nutricional, Campinas, SP, v. 16, n. 2, p. 1-19, 2009.

SEGALL-CORRÊA, A. M. et al. Projeto: acompanhamento e avaliação da segurança alimentar de famílias brasileiras: validação de metodologia e de instrumento de coleta de informação. Relatório Técnico. Campinas: Unicamp, 2003.

SILVA, N. N. Amostragem probabilística. 2. ed. São Paulo: EDUSP, 2001.

SOARES, S.; SÁTYRO, N. O Programa Bolsa Família: desenho institucional, impactos e possibilidades futuras. Brasília: IPEA, 2009.

TRALDI, D. R. C. et al. Repercussões do Programa Bolsa Família no município de Araraquara, SP: um olhar sobre a segurança alimentar e nutricional dos beneficiários. Interações, Campo Grande, MS, v. 13, n. 1, p. 23-37, jan./jun. 2012.

VAITSMAN, J.; ANDRADE, G. R. B.; FARIAS, L. O. Proteção social no Brasil: o que mudou na assistência social após a Constituição de 1988. Revista Ciência \& Saúde Coletiva, Rio de Janeiro, v. 14, n. 30, p. 731-41, 2009.

\section{Sobre autores:}

André Souza Suzart: Mestre em Saúde Pública pela Escola Nacional de Saúde Pública Sergio Arouca (ENSP) da Fundação Oswaldo Cruz (FIOCRUZ). Professor substituto da Universidade Federal do Vale do São Francisco (UNIVASF). E-mail: andre_suzart@hotmail.com

Aldo Pacheco Ferreira: Doutor em Engenharia Biomédica pela Universidade Federal do Rio de Janeiro (UFRJ). Professor do Programa de Pós-Graduação em Saúde Pública pela Escola Nacional de Saúde Pública Sergio Arouca (ENSP) da Fundação Oswaldo Cruz (FIOCRUZ) na área de Direitos humanos e Saúde. E-mail: aldopachecoferreira@gmail.com 
